\title{
ORIGAMI FABRICATION OF THREE-DIMENSIONAL BIOFUEL CELLS WITH NOVEL ANODE MATERIALS
}

\author{
M. Mohammadifar ${ }^{1}$, J. Zhang ${ }^{2}, O$. Sadik ${ }^{2,3}$, and S. Choi ${ }^{1,3 *}$ \\ ${ }^{1}$ Department of Electrical \& Computer Engineering, State University of New York-Binghamton, NY, USA \\ ${ }^{2}$ Department of Chemistry, State University of New York-Binghamton, NY, USA \\ ${ }^{3}$ Center for Research in Advanced Sensing Technologies \& Environmental Sustainability (CREATES), \\ State University of New York-Binghamton, NY, USA
}

\begin{abstract}
We demonstrated a novel origami technique for fabricating three-dimensionally structured microbial fuel cells (MFCs) by using paper as a substrate. A simple 3-D MFC was created from a 2-D sheet of paper having four functional compartments (i.e. anode, reservoir, proton exchange membrane (PEM), and aircathode) through high degrees of folding along pre-defined creases. The device rapidly generated power with a small amount of bacteria-containing liquid through rapid adsorption and attachment of the bacterial cells to the anode. Graphite-polymer composite and graphite ink with activated carbon were readily applicable as novel anodic materials on paper and generated enhanced performance than the conventional graphite ink or gold anode by a factor of 12fold or 4-fold, respectively.
\end{abstract}

\section{INTRODUCTION}

Biomass can be an excellent energy harvesting source that is readily available even in resource-limited environments [1]. Typically, microbial fuel cells (MFCs) have been used as energy transducers that convert the biological energy in biomass directly into electricity via microbial metabolism, and, as such, MFCs are gaining acceptance as a future alternative green energy technology and energy-efficient wastewater treatment method [2-4]. MFCs are comprised of anodic and cathodic compartments separated by a proton exchange membrane (PEM) so that only protons or other cations can travel from the anode to the cathode. A load connects the two electrodes to complete the external circuit. Microorganisms oxidize organic matter in biomass, completing respiration by transferring electrons to the anode. During this reaction, chemical energy is captured in the formation of adenosine triphosphate (ATP) throughout the electron transport chain. With the successful validation of the conceptual macro-sized MFCs as a low-cost renewable energy technology, recent research has focused on miniaturizing MFCs for potentially powering point-of-care (POC) diagnostic tools due to low-cost, environmentally friendly features and easy accessibility in those resource-limited regions [57]. However, the promise of this technology has not yet been translated into practical POC applications, because (i) even smallscale MFCs require a relatively high start-up time to accumulate and acclimate microorganisms on the anodic surface (several hours to days), (ii) their device configuration is complicated with necessary multifunctional parts (anode, cathode, and ion exchange membrane) along with microfluidic tubing for liquid inlets/outlets, and (iii) their operation requires additional power/equipment to continuously inject organic fuels.

In this work, we reported a novel origami technique for fabricating three-dimensionally structured MFCs by using flexible paper substrate (Fig 1). A 3-D MFC was directly created from a 2$\mathrm{D}$ sheet of paper through high degrees of folding along pre-defined creases (Fig 1a 1d). The device included four functional layers; (1) anode, (2) reservoir, (3) proton exchange membrane (PEM), and (4) air-cathode. This fabrication/material breakthrough allowed the creation of a novel MFC platform made only of paper
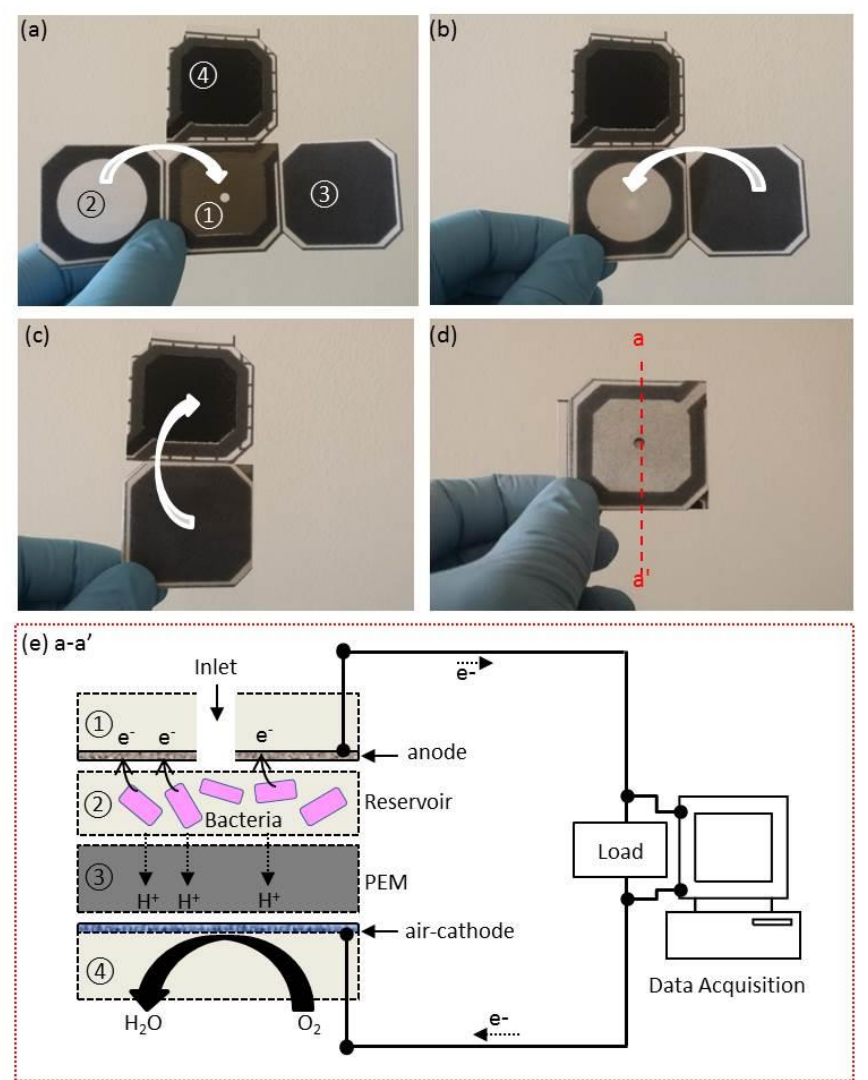

Figure 1: (a) (d) Origami fabrication of the paper-based $M F C$ and (e) schematic diagram of a cross section of the device and its test setup.(1) anode, (2) reservoir, (3) PEM, and (4) air-cathode).

substrates unlike our previous carbon cloth-based paper devices [810]. The MFC configuration/operation became simplified by using paper because the microfluidic reservoir/PEM were easily patterned with hydrophobic wax and the device had the ability to flow the fluid through capillary action without external pump/tubing. In addition, the device rapidly generated power with a small amount of bacteria-containing liquid through rapid adsorption and attachment of the bacterial cells to the anode (Fig 1e). The device platform developed in this work also provided a simple technique to explore anodic materials on a paper substrate. Graphite-polymer composite and graphite ink with activated carbon were readily applicable as a novel anodic material on paper and generated enhanced performances than the conventional graphite ink or gold anode. This work will represent the fusion of the art of origami and paper-based MFC technology, which could provide a paradigm shift in the architecture, material, and design of integrable and stackable paper-based batteries. 


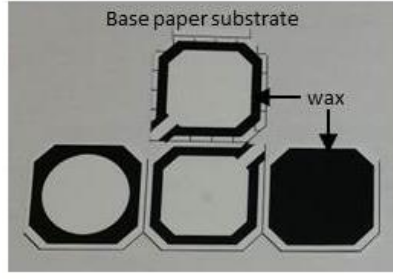

(a) Print wax

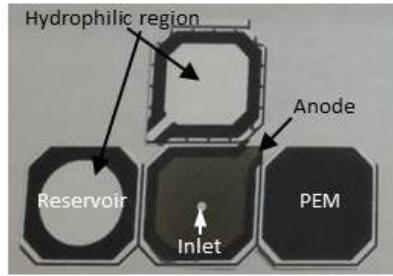

(c) Put anode materials

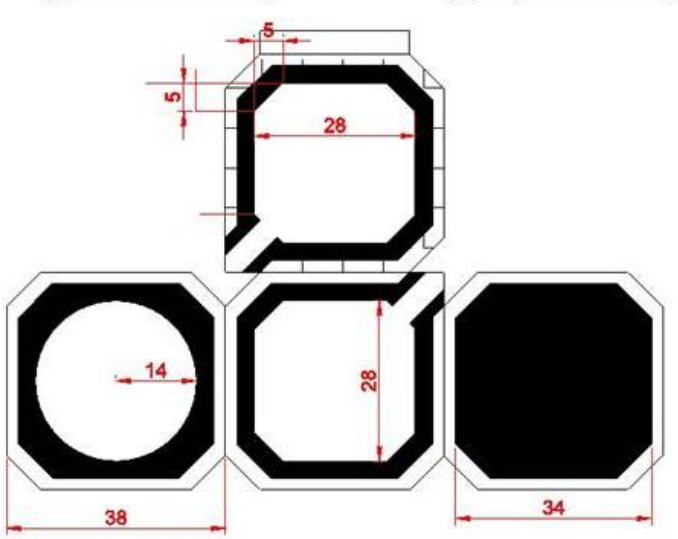

(e) Device dimension

Figure 2: (a) (d) Fabrication processes of the paper-based $M F C$ and (e) the device size (mm). Six devices were prepared with different anodic materials.

\section{EXPERIMENTAL SET-UP}

Device Fabrication and operating principle

The 2-D sheet of paper (Whatman \#1 filter paper) was designed and patterned to have folding tabs on which the anode, reservoir, PEM, and air-cathode were functionalized, respectively (Fig 2). The hydrophilic zones were first prepared with hydrophobic wax boundaries (Fig 2a). The right tab patterned with melted wax was used as the PEM, providing hydrophobic properties and proton conductance (Fig 2b). An anodic material was applied on the middle tab with an inlet hole for bacterial injection (Fig 2c). The left tab was used as a paper reservoir to contain bacterial cells and their organic fuels (Fig 2d). An activate carbon (AC) based air-cathode was constructed on the top tab of the paper. Anode/reservoir/PEM/air-cathode sandwiched construct was well aligned by folding the tabs along pre-defined creases (Fig. 1). The bacterial cells oxidized the organic media in the reservoir layer, completing respiration by transferring electrons to the anode. The protons produced during the microbial metabolism diffused across the hydrophobic wax-based PEM and reduced to water in the presence of oxygen at the air-cathode.

\section{Anode preparation on paper}

We prepared five different anode materials for the paper-based MFC. Four different anode materials were screen-printed on the hydrophilically-patterned paper zone with the surface area of 7.34 $\mathrm{cm}^{2}$ (Fig 2e); (i) composite graphite particle/PAA (Polyacrylic acid), (ii) composite graphite particle $/ 40 \%$ PTFE (Polytetrafluoroethylene) (iii) graphite ink with $\mathrm{AC}$, and (iv) graphite ink alone. The graphite ink (Ercon Inc.) has been widely used as a common electrode material for paper-based devices [11]. The additive polymers such as PAA and PTFE can be very useful as an anode material due to their facile processibility, hydrophilicity, biocompatibility and stability, all of which lead to a high bacteria density with high biodiversity while the carbon materials increase the mechanical properties and electrocatalytic activities $[12,13]$. Graphite particle (particle size distribution: $0.2 \mu \mathrm{m} \sim 20 \mu \mathrm{m})$ and $\mathrm{AC}$ can create activation centers on anodic surfaces to improve the bacterial adhesion/biofilm formation and the electron transfer efficiencies [14]. (v) The fifth anode was prepared by depositing $100 \mathrm{~nm}$ of gold $(\mathrm{Au})$ on the substrate with chrome as the adhesion layer. Since gold is one of the most standard biocompatible materials for MEMS structures, many micro-sized MFCs use gold as an electrode material [15].

\section{Air-cathode preparation on paper}

AC-based air-cathode was first constructed on one side of the paper with carbon spray to provide structural support and to function as a current collector [16]. Then, the AC catalysts (CABOT Corporation) $\left(15 \mathrm{mg} / \mathrm{cm}^{2}\right)$ with a blinder solution was applied on top of the carbon-sprayed paper zone, which was subsequently air-dried for $24 \mathrm{~h}$. The blinder solution was prepared by adding a mixture of (i) $1200 \mu \mathrm{L}$ of $5 \mathrm{wt} \%$ Nafion solution, (ii) $150 \mu \mathrm{L}$ of DI water, and (iii) $600 \mu \mathrm{L}$ of isopropanol into a beaker, followed by ultrasonication for $1 \mathrm{~min}$. Due to the porous structure of the paper, the other side of the paper allowed the oxygen to reach carbon to complete the redox reaction for the MFC operation [17].

\section{Inoculum and measurement setup}

Shewanella oneidensis MR-1 were grown from $-80^{\circ} \mathrm{C}$ glycerol stock cultures by inoculating $20 \mathrm{~mL}$ of L-broth medium with gentle shaking in air for $24 \mathrm{~h}$ at $35^{\circ} \mathrm{C}$. The L-broth media consisted of $10.0 \mathrm{~g}$ tryptone, $5.0 \mathrm{~g}$ yeast extract and $5.0 \mathrm{~g} \mathrm{NaCl}$ per liter. Both cultures were then centrifuged at $5,000 \mathrm{rpm}$ for $5 \mathrm{~min}$ to remove the supernatant. The bacterial cells were re-suspended in a new medium and used as an anolyte for the device. We measured the potentials between the anodes and the cathodes with a data acquisition system (National Instrument, USB-6212), and recorded the readings every $1 \mathrm{~min}$ via a customized LabView interface. An external resistor connected between the anode and the cathode closed the circuit. The current through this resistor was calculated using Ohm's law.

\section{Bacterial Fixation and SEM Imaging}

The paper-based MFCs were disassembled, rinsed, and adherent bacteria on each anode were immediately fixed in $2 \%$ glutaraldehyde solution overnight at $4^{\circ} \mathrm{C}$. Samples were then dehydrated by serial 5 min transfers through $50,70,80,90,95$, and $100 \%$ ethanol. Fixed samples were examined using an FESEM (Field Emission SEM) (Supra 55 VP, Zeiss).

\section{RESULTS AND DISCUSSION}

After the inoculum was dropped through the inlet of the device, the voltage curves with and without the load were measured (Fig 3a). Before closing the MFC circuits with resistors, the open circuit voltages were recorded for 8 minutes. Then $100 \mathrm{k} \Omega$ and subsequently $10 \mathrm{k} \Omega$ was connected to enable current generation. 

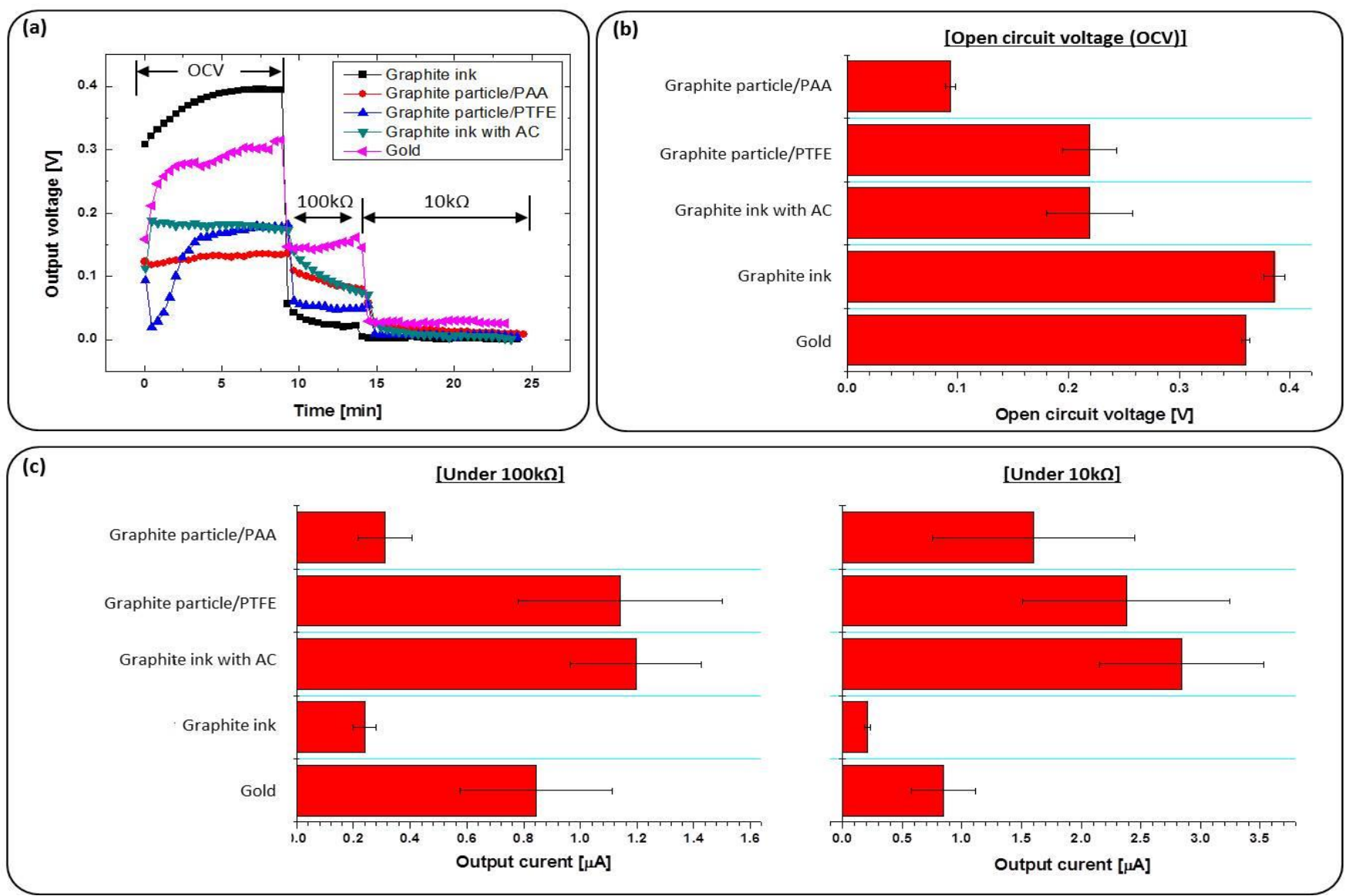

Figure 3: (a) Voltage profiles measured from the devices with six different anodic materials. (b) Open circuit voltages were measured for the first 8 minutes and then all devices were connected to (c) $100 \mathrm{k} \Omega$ and (d) $10 \mathrm{k} \Omega$ external loads and the currents were calculated. (Poly(amic) acid: PAA, Polytetrafluoroethylene: PTFE)

\section{Open circuit voltages (OCVs)}

Fig $3 b$ shows the OCVs of the MFCs with different anodic materials; graphite particle/PAA, graphite particle/PTFE, graphite ink with AC, graphite ink and gold. The OCVs increased and reached saturated values, which were relatively less than that of conventional MFCs (Fig 3a). Oxygen penetration into the anode abiotically and biotically reacted with the anode and reduced the OCVs [18]. Measured OCVs varied between the different MFCs, clearly indicating performance variations according to the anodic materials. The composite anodic materials such as graphite particle/PAA, graphite particle/PTFE, and graphite ink with AC generated the lower OCVs than the graphite ink and gold.

\section{Current generation}

After reaching an approximately stable OCV, we connected a $100 \mathrm{~K} \Omega$ resistor between the anode and cathode electrodes (Fig 3c). After 5 minutes of operation (Fig 3a), the resistor was replaced with the $10 \mathrm{k} \Omega$ to harvest more current generation. The highest current was obtained by the graphite ink with $\mathrm{AC}$ as an anode, which was twelve times higher magnitude than that of the graphite ink only. This result indicates that AC particles provide a larger bio-accessible surface area and higher electron transfer efficiency than on the graphite ink-based anode. The graphite particles with PTFE also showed a good performance in current generation. The PTFE has long been used as a binder material for fabricating gas diffusion electrodes in fuel cells because of its chemical stability and hydrophobic nature [19]. Furthermore, the use of PTFE leads to a more porous structure which can improve the surface area and the affinity of the anode electrode for the bacterial cells' attachment [20]. The hydrophilic property of the material also contributes to the higher performance of the MFC. However, the higher hydrophilic features, the thicker and more condensed bacterial biofilm may be formed, clogging the pores and consequently hindering the transport of nutrients/waste products. Moreover, incorporation of PTFE can decrease the electric conductivity of the electrode [20]. Therefore, the concentration of PTFE must be optimized to maximize the performance. In this work, $40 \%$ PTFE with the graphite particles generated the best performance.

Although the MFC with the graphite particle/PAA showed lower current production than the other composite materials, the current generation gradually increased with time under both resistors (Fig 3a). This is mainly due to the fact that the introduction of PAA can increase the hydrophilicity of the anode and provides better bacterial attachment with time. The best performance was obtained by the $60 \%$ PAA with the graphite particles. The gold material showed relatively higher current under the $100 \mathrm{k} \Omega$ resistor, but their performance significantly degraded with the $10 \mathrm{k} \Omega$. Although the gold is one of the most standard biocompatible materials for bio-devices, it is not appropriate for the disposable, inexpensive paper-based device applications. Overall, the maximum current generation $(3 \mu \mathrm{A})$ was obtained from the graphite ink with AC followed by graphite particle/PTFE 


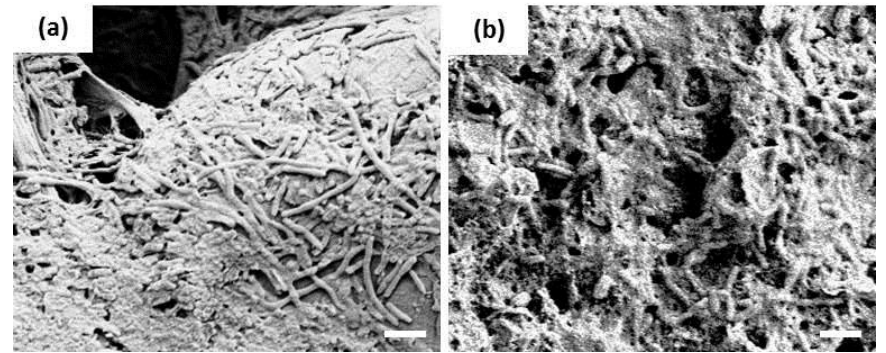

Figure 4: SEM images of the (a) reservoir and (b) anode (graphite ink with AC) used in the paper-based MFC. Scale bar is $2 \mu \mathrm{m}$.

composite, which was much higher than the conventional graphite ink-based anode on paper. Very densely packed bacterial cells were observed on the paper reservoir and on the graphite ink/AC anode after current generation (Fig. 4). The strong capillary force of the paper reservoir contributed to rapid adsorption of the inoculum and promoted aggregates of bacterial cells in the paper matrix, allowing a number of cells to attach to the anode.

\section{CONCLUSION}

This work provided a novel technique for manufacturing 3-D MFCs by using paper as a substrate and applying the art of origami. Moreover, we investigated the several composite materials to optimize surface characteristics for bacterial attachment and electron transfer efficiencies. The carbon-PTFE composite and the graphite ink/AC showed the potential as a novel anode material for paper-based MFCs to increase surface area, porosity, biocompatibility, conductivity and biofilm formation. In particular, the graphite ink/AC material outperformed conventional graphite ink by a factor of 12-fold and even biocompatible gold electrode by 4 -fold.

\section{ACKNOLOWDGMENT}

This work is supported by NSF (ECCS \#1503462) and NSF (IOS \#1543944).

\section{REFERENCES}

[1] B.E. Rittmann, "Opportunities for Renewable bioenergy using microorganisms", Biotechnology and Bioengineering, 100, 203-212 (2008).

[2] D.R. Lovley, "Electromicrobiology", Annu. Rev. Microbiol., 66, 391-409 (2012).

[3] V.B. Oliveira, M. Simoes, L.F. Melo, and A.M.F.R. Pinto, "Overview on the developments of microbial fuel cells", Biochemical Engineering Journal, 73, 53-64 (2013).

[4] J. Babauta, R. Renslow, Z. Lewandowski, and H. Beyenal, "Electrochemically active biofilms: facts and fiction", Biofouling: The journal of Bioadhesion and Biofilm Research, 28, 789-812 (2013).

[5] S. Choi, "Microscale microbial fuel cells: advances and challenges", Biosensors and Bioelectronics, 69, 8-25 (2015).

[6] C. Siu, and M. Chiao, "A microfabricated PDMS microbial fuel cell," J. Microelectromechanical Systems, 17, 1329-1341 (2008)

[7] H. Lee and S. Choi, "A micro-sized biosolar cell for selfsustaining power generation”, Lab Chip, 15, 391-398 (2015).

[8] A. Fraiwan, S. Mukherjee, S. Sundermier, H.-S. Lee and S. Choi, "A paper-based Microbial Fuel Cell: Instant battery for disposable diagnostic devices", Biosensors and Bioelectronics, 49, 410-414 (2013).
[9] A. Fraiwan, H. Lee and S. Choi, "A multi-Anode paper-based microbial fuel cell: A potential power source for disposable biosensors", IEEE Sensors Journal, 14, 3385-3390 (2014).

[10] A. Fraiwan, and S. Choi, "Bacteria-Powered Battery on Paper", Physical Chemistry Chemical Physics, 16, 2628826293 (2014).

[11] D.D. Liana, B. Raguse, J.J. Gooding, and E. Chow, "Recent advances in paper-based sensors", Sensors, 12, 11505-11526 (2012).

[12] M. Zhou, M. Chi, J. Luo, H. He, and T. Jin, "An overview of electrode materials in microbial fuel cells", Journal of Power Source, 196, 4427-4435 (2011).

[13] M. Ghasemi, W.R.W. Daud, S.H.A. Hassan, S. Oh, M. Ismail, M. Rahimnejad, and J. Md Jahim, "Nano-structured carbon as electrode material in microbial fuel cells: A comprehensive review", Journal of Alloys and Compounds, 580, 245-255 (2013).

[14] V. Gadhamshetty, and N. Koratkar, "Nano-engineered biocatalyst-electrode structures for next generation microbial fuel cells", Nano Energy, 1, 3-5 (2012).

[15] S. Mukherjee, S. Su, W. Panmanee, R.T. Irvin, D.J. Hassett, and S. Choi, "A microliter-scale microbial fuel cell array for bacterial electrogenic screening", Sensors and Actuators: A. Physical, 201, 532-537 (2013).

[16] X. Zhang, X. Xia, I. Ivanov, X. Huang, and B.E. Logan, "Enhanced activated carbon cathode performance for microbial fuel cell by blending carbon black", Environ. Sci. Technol., 48, 2075-2081 (2014).

[17] H. Lee, and S. Choi, "An origami paper-based bacteriapowered battery”, Nano Energy, 15, 549-557 (2015).

[18] S. Choi, H.-S. Lee, Y. Yang, P. Parameswaran, C.I. Torres, B.E. Rittmann and J. Chae, "A $\mu$ L-scale Micromachined Microbial Fuel Cell Having High Power Density", Lab on a Chip, 11, 1110-1117 (2011).

[19] S. Litster, and G. McLean, "PEM fuel cell electrodes", J. Power Sources, 130, 61-76 (2004).

[20] T. Zhang, Y. Zeng, S. Chen, X. Ai, and H. Yang, "Improved performances of E. coli-catalyzed microbial fuel cells with composite graphite/PTFE anodes", Electrochemistry Communications, 9, 349-353 (2007).

\section{CONTACT}

*S. Choi, tel: +1-607-777-5913; sechoi@binghamton.edu 